\title{
Thrombophlebitis der Vena basilica nach beidseitiger Brustaugmentation
}

\author{
Rau, Monika ; Kaiser, Robert ; Knechtle, Beat
}

\begin{abstract}
We report the case of a 33 years old woman who suffered from pain in the left upper arm and a lack of sensibility in the inner part of the left hand 14 days after having had a breast augmentation. We found an increase in d-dimers. Otherwise blood results were normal. Duplex sonography showed a thrombophlebitis of the left vena basilic vein. The patient was treated with Rivaroxaban (Xarelto ${ }^{\circledR}$ ) $2 \times 15 \mathrm{mg}$ per day for four weeks and had to wear a compression bandage. Due to the treatment, pain relief set in soon. The basilica vein was recanalized five weeks after diagnosis.
\end{abstract}

DOI: https://doi.org/10.1024/1661-8157/a002708

Other titles: Thrombophlebitis of the basilic vein after bilateral breast augmentation

Posted at the Zurich Open Repository and Archive, University of Zurich

ZORA URL: https://doi.org/10.5167/uzh-138556

Journal Article

Accepted Version

Originally published at:

Rau, Monika; Kaiser, Robert; Knechtle, Beat (2017). Thrombophlebitis der Vena basilica nach beidseitiger Brustaugmentation. Praxis, 106(13):723-726.

DOI: https://doi.org/10.1024/1661-8157/a002708 
${ }^{1}$ Gesundheitszentrum St. Gallen, ${ }^{2}$ Gefässmedizin Klinik Stephanshorn

Monika Rau ${ }^{1}$, Robert Kaiser ${ }^{2}$, Beat Knechtle ${ }^{1}$

\section{Thrombophlebitis der Vena basilica nach beidseitiger}

Brustaugmentationsoperation

Thrombophlebitis of vena basilica after bilateral breast augmentation surgery 
Im Artikel verwendete Abkürzungen

COPD Chronic obstructive pulmonary disease

CRP C-reaktives Protein 


\section{Anamnese und Befunde}

Die 33-jährige Mutter zweier Kinder unterzieht sich im Ausland dem Wahleingriff einer Brustaugmentationsoperation beidseits. Die Operation verläuft ohne Komplikationen. Die postoperativen Wundkontrollen bei der Hausärztin zeigen keine Besonderheiten, die Blutwerte (Routinehämatologie und CRP) waren normal. Zwei Wochen postoperativ stellt sich die Patientin aber mit seit 3 Tagen andauernden Schmerzen im Oberarm links und Sensibilitätseinschränkungen an der linken Hand ulnarseits gelegen bei der Hausärztin vor.

Im Status zeigt sich ein deutlicher Druckschmerz im linken Oberarm medial mit starker Schwellung und Induration sowie Dysästhesien im Verlauf des $n$. ulnaris links. Im Praxislabor zeigte sich ein erhöhtes D-Dimer von $3.9 \mu \mathrm{g} / \mathrm{ml}$ (Referenz $<0.5 \mu \mathrm{g} / \mathrm{ml}$ ) sowie ein nicht erhöhtes C-reaktives Protein (CRP) von $3.1 \mathrm{mg} / \mathrm{l}$ (Referenz $<5.0 \mathrm{mg} / \mathrm{l}$ ). Als Nebendiagnosen liegt eine COPD bei Nikotinabusus vor, wobei die Patientin vor kurzem mit dem Rauchen aufgehört hat.

\section{Differentialdiagnostische Überlegungen}

Aufgrund der Schmerzen und der Schwellung im linken Oberarm konnte ein entzündliches resp. infektiöses Geschehen nicht ausgeschlossen werden, die Druckdolenz liess an eine Entzündung denken, auch eine Thrombose musste in Betracht gezogen werden. Vor allem die Dysästhesien im Verlauf des $n$. ulnaris waren a priori nicht erklärbar. Die Haut war nicht gerötet, nicht überwärmt. Bei erhöhten D-Dimeren musste ein intravasaler Prozess, eine Thrombose resp. Thrombophlebitis der Oberarmgefässe in Betracht gezogen werden.

\section{Weitere Abklärungsschritte und Verlauf}

Die gleichentags notfallmässig durchgeführte apparativ-technische gefässmedizinische Untersuchung zeigte eine deutlich dilatierte und nicht komprimierbare $V$. basilica des linken Oberarmes. In der farbcodierte Duplexsonographie fand sich eine ausgeprägte Thrombophlebitis der $V$. basilica links bis zur Einmündung in die $V$. brachialis und bis in die Höhe der Axilla reichend (Abbildungen 1 und 2), ohne Thrombose der $V$. brachialis links (Abbildung 3). 
Aufgrund der nachgewiesenen Thrombophlebitis à chaude bis zur Mündung reichend wurde eine orale Antikoagulation mit Rivaroxaban (Xarelto ${ }^{\circledR}$ ) 2 × 15 mg eingeleitet, um die Propagation des thrombotischen bzw. phlebitischen Prozesses mit konsekutiver Thrombose der $V$. brachialis bzw. Verschleppung von Thromben zu verhindern. Zudem wurde ein Oberarmkompressionsstrumpf appliziert.

Im weiteren Verlauf zeigte sich 5 Wochen nach Diagnose im Rahmen der gefässmedizinischen Nachkontrolle eine rekanalisierte $V$. basilica links (Abbildung 4) mit gering postphlebitisch veränderter Wand aber freier Perfusion. Auch sonst lag kein Hinweis auf eine Thrombophlebitis oder Thrombose vor. Die Beschwerden der Patientin waren komplett regredient. Die Einnahme von Rivaroxaban (Xarelto $\left.{ }^{\circledR}\right)$ und das Tragen des Stützstrumpfes konnte beendet werden.

\section{Diagnose}

Thrombophlebitis der $V$. basilica links 12 Tage nach Narkose mit vascular access bei Wahleingriff einer beidseitigen Brustaugmentation

\section{Kommentar}

In diesem Fallbericht beschreiben wir eine iatrogene Komplikation einer Thrombophlebitis an der oberen Extremität nach einer beidseitigen Brustaugmentationsoperation, assoziiert mit dem intravenösen Zugang am linken Arm intraoperativ. Da die Operation im Ausland durchgeführt wurde konnten wir den Operationsbericht und das Anästhesieprotokoll nicht einsehen.

Eine Thrombophlebitis ist eine akute Entzündung und Thrombose einer oberflächlichen Vene. Besonders häufig treten Thrombophlebitiden bei Krampfadern auf. Klinisch imponieren bei einer floriden Thrombophlebitis Schmerzen im Bereich der entzündeten Vene, eine Rötung und Überwärmung und ein Ödem sowie eine Induration in der Umgebung. Im vorliegenden Fall lagen ein Druckschmerz, eine Schwellung und eine Induration vor, hingegen keine klassischen Zeichen einer Thrombophlebitis wie Rötung und Überwärmung, da die $V$. basilica am Oberarm subfascial gelegen ist. 
Die Thrombophlebitis wurde in der Vergangenheit lange unterschätzt und als harmlos beurteilt. Heute wissen wir, dass sich die Entzündung in das tiefe Venensystem propagieren und so zu Komplikationen führen kann. Deshalb musste im aktuellen Fall ultrasonographisch eine Thrombose bzw. eine Thrombuspropagation ausgeschlossen werden.

Bei der Überlegung ob eher ein entzündlich-infektiöses Geschehen oder eine Thrombose vorliegt helfen die D-Dimere nicht viel weiter, denn sie können in beiden Situationen positiv sein. Das Verhalten des CRP könnte in so einer Situation hilfreich sein. Allerdings ist bekannt dass CRP und D-Dimer im Falle einer tiefen Venenthrombose erhöht sind. Die Bestimmung des CRP bei Verdacht auf eine tiefe Venenthrombose ist nicht hilfreich in Diagnosestellung und kann auch keine Entscheidung liefern ob es eher ein thrombotisches oder ein entzündliches Geschehen oder beides ist [1].

\section{Gründe für Thrombophlebitiden der Oberarmgefässe}

Die Ursachen der meisten Fälle von oberflächlichen Venenentzündungen sind venöse Zugänge insbesondere Verweilkanülen oder Katheter. Bei der Entstehung einer Thrombose resp. Thrombophlebitis gilt noch immer die schon lange bekannte Virchow-Trias mit Endothelalterationen, Veränderungen der Strömungsgeschwindigkeit sowie Veränderungen der Viskosität des Blutes. Tabelle 1 die möglichen generellen Ursachen für eine Thrombophlebitis.

In unserem Fall sind folgende Ursachen in Betracht zu ziehen: mechanische Einwirkung durch lokalen Druck (Druck durch Lagerung, Punktion oder eine Blutdruckmanschette), Reizung der Gefässinnenwand durch die Venenverweilkanüle, Punktionszugang im bewegten Bereich sowie die Gabe von Medikamenten mit kristalloiden Anteilen, wobei möglicherweise eine Substanz auch nicht korrekt aufgelöst wurde. Eine akute Entzündung der Venenwand durch mechanische oder chemische Reizung - etwa durch einen peripheren Venenkatheter oder ein Endothelschaden durch kristalloide Substanzen, wie etwa Ampicillin oder Carbamazepin - sowie die Einschwemmung von Keimen wird angenommen [2-5]. Auch eine Freisetzung von entzündlichen Mediatoren aus einem Blutgerinnsel wird diskutiert [5]. Eine hormonelle Genese durch Antikonzeptiva kommt neben einer mechanischen Einwirkung z.B. einem Liegetrauma auch in Frage.

Tabelle 2 stellt die möglichen Differentialdiagnosen zusammen wenn klinisch der Verdacht auf eine Thrombophlebitis vorliegt. In dieser Liste sind auch Erkrankungen geführt an die man denken muss wenn eine unklare Ursache für eine Thrombophlebitis vorliegt. 


\section{Gründe für assoziierte neurologische Beschwerden}

Bei der Patientin lagen auch noch Sensibilitätsstörungen im Ausbreitungsgebiet des $n$. ulnaris vor. Die Nervenmitbeteiligung machte sich durch ein Taubheitsgefühl im kleinen Finger und an der äusseren Seite des Ringfingers und im angrenzenden Handtellerbereich des entsprechenden Armes bemerkbar. In unserem Falle war das Perineurium in den entzündlichen Prozess durch die anatomische Nähe zur V. basilica involviert, was die Dysästhesien erklärt. Die Dysästhesien verschwanden sukzessive mit dem Rückgang des entzündlichen Prozesses und der Thrombophlebitis.

Solche mit Venenverweilkanülen assoziierten Ereignisse sind keine Seltenheit, werden jedoch oft unzulänglich dokumentiert und verifiziert.

\section{Praktische Bedeutung aus hausärztlicher Sicht}

Eine Thrombophlebitis einer Oberarmvene als postoperative Komplikation ist per se nicht häufig. Nicht unerwähnt bleiben sollten in diesem Fall die möglichen qualitativen Defizite bei Behandlungstourismus. Immer mehr Patienten zieht es ins Ausland, um sich dort medizinisch behandeln zu lassen. Ob Zahnersatz in Polen, eine Erholungskur in Tschechien oder eine Augenoperation in Ungarn: Wer den Arztbesuch jenseits der Grenzen erwägt, sollte sich möglicher qualitativer Mängel bewusst sein. Insbesondere bei nichtkassenpflichtigen Eingriffen sollte sich der/die Patient/in vor einem möglichen Eingriff im Ausland gut über mögliche Risiken und Komplikationen aufklären und im Falle einer Komplikation wissen wie er/sie sich zu verhalten hat. 


\section{Key messages}

Auch eine an und für sich komplikationsarme resp. komplikationslose Operation kann mit perioperativen Komplikationen assoziiert sein

Bei einer Schwellung mit Druckdolenz nach vascular access im Oberarm mit/ohne Dysästhesien sollte eine Blutanalyse mit D-Dimeren durchgeführt werden und eine Duplexsonografie zum Ausschluss einer Thrombophlebitis.

Es ist bekannt, dass kristalloide Substanzen wie Antibiotika Entzündungen der Gefässinnenwand verursachen können. Da auch Sekundärkomplikationen wie eine Spondylodiscitis durch Infekte durch Verweilkanülen beschrieben sind, sollte und muss eine Dokumentation der Kanülen und möglicher Komplikationen erfolgen und frühzeitig eine interdisziplinäre Behandlung eingeleitet werden. 


\section{Zusammenfassung}

Wir berichten über eine 33 jährige Patientin, die sich 14 Tage nach BrustaugmentationsOperation mit Schmerzen im linken Oberarm und mit Sensibilitätseinschränkungen in der Handinnenseite links in der hausärztlichen Praxis vorstellt. Im Labor fanden sich die erhöhten D-Dimere. In der Duplexsonographie lag eine ausgeprägte Thrombophlebitis der v. basilica links vor. Nach Gabe von Rivaroxaban (Xarelto ${ }^{\circledR}$ ) für vier Wochen und des Tragens des Kompressionsverbandes kam es zu einer Regredienz der Beschwerden. Fünf Wochen nach Diagnosestellung war die $V$. basilica links wieder rekanalisiert.

\section{Schlüsselwörter}

Operation; Komplikation; postoperativ; Thrombose; Prophylaxe 


\section{Summary}

We report the case of a 33-years-old woman, who suffered from pain in the left upper limb and a lack of sensibility in the inner part of the left hand 14 days after having had a breast augmentation operation. We found an increase in d-dimers, besides oft that the blood results were normal. The duplex sonography showed a thrombophlebitis of the left vena basilica. The patient was treated with Rivaroxaban (Xarelto $\left.{ }^{\circledR}\right) 2 \times 15 \mathrm{mg}$ per day for four weeks and she had to wear a compression bandage. Due to the treatment pain relief was felt soon. The vena basilica was recanalised six weeks after diagnosis.

\section{Key words}

Surgery; Complication; Postoperative; Thrombosis; Prophylaxis 


\section{Korrespondenzadresse}

Prof. Dr. med. Beat Knechtle

Facharzt FMH für Allgemeinmedizin

Gesundheitszentrum St. Gallen

Vadianstrasse 26

9001 St. Gallen

Telefon $\quad+41(0) 712269300$

Telefax $\quad+41(0) 712269301$

E-Mail beat.knechtle@hispeed.ch 


\section{Literaturverzeichnis}

1 Bucek RA, Reiter M, Quehenberger P, Minar E. C-reactive protein in the diagnosis of deep vein thrombosis. Br J Haematol. 2002 Nov;119(2):385-9.

2 Idvall E, Gunningberg L. Evidence for elective replacement of peripheral intravenous catheter to prevent thrombophlebitis: a systematic review. J Adv Nurs. 2006 Sep;55(6):715-22.

3 Cicolini G, Bonghi AP, Di Labio L, Di Mascio R. Position of peripheral venous cannulae and the incidence of thrombophlebitis: an observational study. J Adv Nurs. 2009 Jun;65(6):1268-73. doi: 10.1111/j.1365-2648.2009.04980.x. Epub 2009 Apr 3.

4 Nishanth S, Sivaram G, Kalayarasan R, Kate V, Ananthakrishnan N. Does elective resiting of intravenous cannulae decrease peripheral thrombophlebitis? A randomized controlled study. Natl Med J India. 2009 Mar-Apr;22(2):60-2.

5 Tagalakis V, Kahn SR, Libman M, Blostein M. The epidemiology of peripheral vein infusion thrombophlebitis: a critical review. Am J Med. 2002 Aug 1;113(2):146-51. 
Stase

Endothelläsion

Gerinnungstörung (Antithrombin III Mangel, Faktor XII Mangel, Protein C und S Mangel)

Paraneoplastisches Syndrom

Rezidiv-Thrombophlebitis

Variko-Phlebitis

Morbus Behcet

Sarkoidose

Morbus Bürger

Tabelle 1: Mögliche Ursachen für eine Thrombophlebitis 
Thrombophlebitis bei Sepsis bei liegendem Venenzugang

Thrombophlebitis nach intravenöser Gabe eines zytotoxischen Medikaments zur Behandlung eines Tumorleidens

Phlebitis saltans bzw. migrans als Folge von Autoimmunerkrankungen/ Gefässerkrankungen wie Wegener-Granulomatose, Lupus erythematodes, Riesenzellarteriitis bei Polymalgia rheumatica, Thrombangitis obliterans Morbus Bürger)

Lyme-Borreliose nach Zeckenbiss

Sweet Syndrom bei myelodysplastischem Syndrom und akuter myeloischer Leukämie

Tabelle 2: Differentialdiagnosen der Thrombophlebitis 


\section{Legende der Abbildungen}

Abbildung 1 Längsschnitt $v$. basilica links mit typischer Thrombophlebitis

Abbildung 2 Querschnitt v. basilica links

Abbildung 3 Atemabhängiger Fluss in v. basilica links und Komprimierbarkeit

Abbildung 4 Rekanalisierte $v$. basilica links (nach 5 Wochen) 